\title{
Heliospheric energetic neutral atoms as a means to determine the anomalous cosmic ray spectrum at the termination shock
}

\author{
A. Czechowski ${ }^{1}$, M. Hilchenbach ${ }^{2}$, and K.C. Hsieh $^{3}$ \\ 1 Space Research Centre, Polish Academy of Sciences, Bartycka 18A, 00716 Warsaw, Poland \\ e-mail: ace@cbk.waw.pl \\ 2 Max-Planck-Institut für Aeronomie, Max-Planck-Strasse 2, 37191 Katlenburg-Lindau, Germany \\ e-mail: hilchenbach@linmpi.mpg.de \\ 3 University of Arizona, Physics Department, AZ 85721 Tucson, USA \\ e-mail: hsieh@physics.arizona.edu
}

Received 4 June 2004 / Accepted 11 October 2004

\begin{abstract}
We demonstrate the possibility of deriving the anomalous cosmic ray (ACR) spectrum at the solar wind termination shock and the strength of the shock using energetic neutral $\mathrm{H}$ and He atoms (ENA) measurements at 1 AU. Assuming that the energetic neutral atoms detected by CELIAS/HSTOF on board SOHO are of ACR origin, we consider $\chi^{2}$ fits to the ACR termination shock spectrum based on approximations to the ACR distribution downstream of the shock. We show that the method becomes much more effective when the low-energy ENA data are combined with the available high-energy ACR data from Voyager spacecraft. The results imply a weak termination shock (compression ratio 2.9).
\end{abstract}

Key words. Sun: solar wind - shock waves - ISM: cosmic rays - interplanetary medium

\section{Introduction}

The recent data from Voyager 1 (Krimigis et al. 2003; McDonald et al. 2003; Burlaga et al. 2003) suggest that the vicinity of the heliospheric termination shock may have been reached. Although the understanding of the results is incomplete, it is possible that the low-energy part of the shockassociated particle spectrum has for the first time become accessible to direct measurement. Interestingly, it also strengthens the case for another, indirect method (Czechowski et al. 1995, 2001a,b; see also Fahr \& Lay 2000) of sensing the shockaccelerated spectrum by means of the energetic neutral atoms (ENA). One point is that the high intensity of the observed spectrum supports the viability of the method (see Hsieh et al. 1992 for estimations of different heliospheric sources of ENA). The other is that the discussion of the interpretation of the new data highlighted the need for understanding the global, largescale structure of the termination shock (e.g. in connection with the idea that the acceleration of low-energy ions occurs at particular favoured locations at the termination shock, Schwadron $\&$ McComas 2003): in this field the remote-sensing methods could offer an important supplement to local measurements by Voyagers.

In the following we consider the question how the ENA observations can be used as a source of information about the distant heliosphere, in particular about the termination shock, and to what extent this is possible without relying on detailed models of the heliosphere. In particular, we present an attempt to estimate the intensity and the spectrum of the anomalous cosmic ray (ACR) ion flux at the termination shock from the flux of ENA measured by the CELIAS/HSTOF: the HighEnergy Suprathermal Time-of-Flight sensor of the Charge, Element, and Isotope Analysis System (Hilchenbach et al. 1998, 2001; Czechowski et al. 2004) combined with the highenergy ACR data from Voyagers (Cummings et al. 2002). To this end we use the approximations valid for the special choices of viewing direction (apex and the heliotail). To connect with the high-energy ACR data we use Steenberg \& Moraal (1999) formula for the ACR spectrum at the termination shock. The energy range we consider is $\sim 10^{1}-10^{2} \mathrm{keV} / \mathrm{n}$. Important information about the termination shock and the heliosphere can also be provided by very low energy $(\leq 1 \mathrm{keV} / \mathrm{n})$ ENA data (Gruntman 1992; Gruntman et al. 2001).

\section{Observations}

Observations of the hydrogen and helium energetic atoms of possibly heliospheric origin come from HSTOF/CELIAS on SOHO (Hilchenbach et al. 1998, 2001). A detailed description of the ENA measurements by HSTOF is given in Hilchenbach et al. (1998). Here we recapitulate some of the most relevant points.

The HSTOF field-of-view covers the sector $4^{\circ}$ wide in the ecliptic plane and $\pm 17^{\circ}$ off the ecliptic. The line-of-sight is 


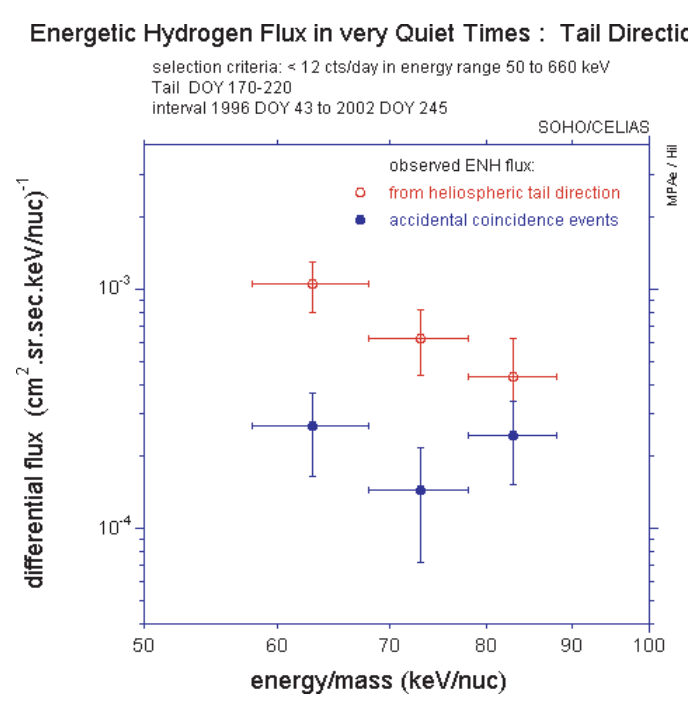

Energetic Hydrogen Flux in very Quiet Times : APEX Direction selection criteria: 12 ctsiday in energy range 50 to $660 \mathrm{keV}$ Apex DoY 1-169, 221-365/366 interval 1996 DOY 43 to 2002 DOY 245

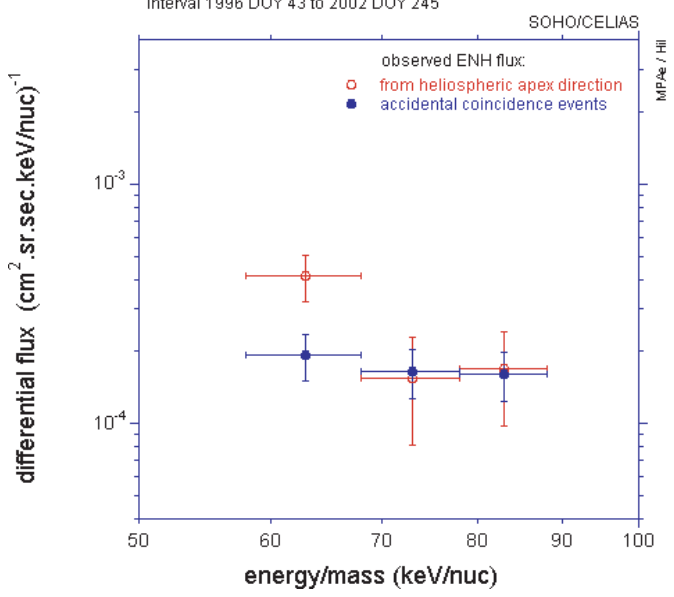

Fig. 1. The energetic neutral hydrogen (ENH) flux from the "heliotail" sector $\left( \pm 25^{\circ}\right.$ from the local ISM anti-apex direction) and from the "apex" sector (the part of the HSTOF field-of-view not included in the heliotail sector) measured by CELIAS/HSTOF ( $1 \sigma$ error bars). The accidental coincidence background is also shown.

directed at $37^{\circ}$ off the sunward direction. In consequence, all directions within $\pm 17^{\circ}$ from the ecliptic are scanned once a year.

Figures 1 and 2 show the energetic neutral hydrogen $(\mathrm{ENH})$ and helium (ENHe) spectrum derived from the HSTOF measurements (Hilchenbach et al. 1998, 2001; Czechowski et al. 2004). Because of low ENA flux intensities the ENA observations are restricted to the "quiet times" corresponding to ion counts being below appropriate upper limits. Only during the first two years of operation (1996 and 1997, near the solar minimum) did the "quiet time" periods cover a large part of the total observation time. As a result, most of the ENA data points come from these two years. The "tail" data are defined as the average flux coming from the sector within $\pm 25^{\circ}$ (in the ecliptic plane) of the LISM anti-apex direction. The "apex" data are the averages over the remaining part of the HSTOF field of view. Note that the measured ENA flux from the "tail" direction is higher than that from the "apex" direction. If the ENA are of ACR origin this feature follows as a consequence of

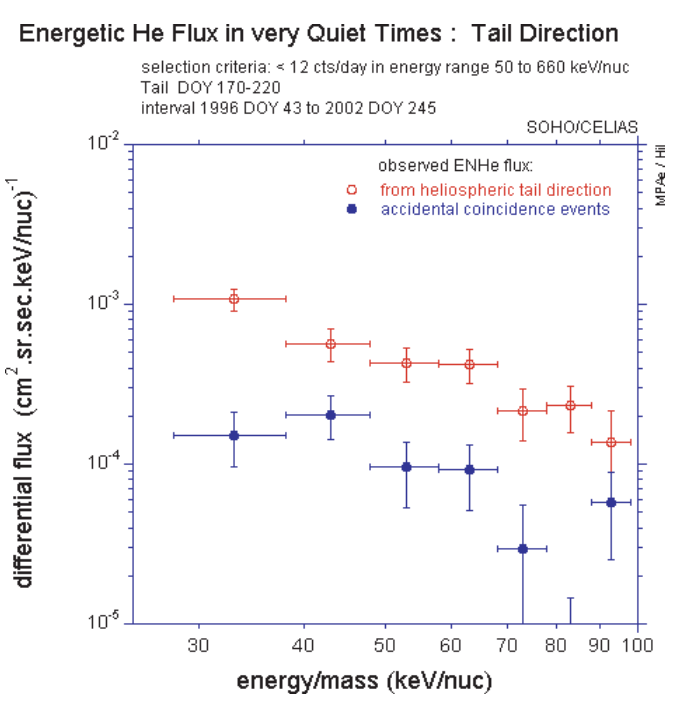

Energetic He Flux in very Quiet Times : Apex Direction Quiet time selection criteria: as ENH Apex DOY 1-169,221-365/366 Interval 1996 DOY 43 to 2002 DOY 245

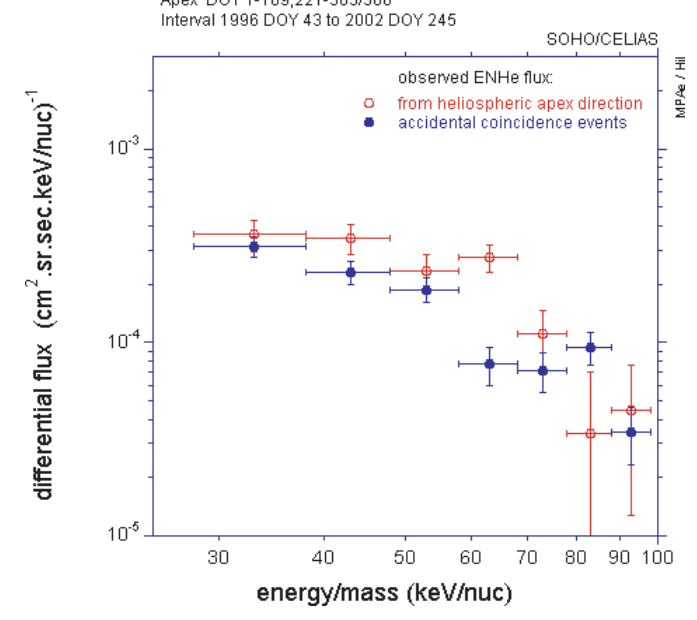

Fig. 2. The energetic neutral helium (ENHe) flux from the "heliotail" sector $\left( \pm 25^{\circ}\right.$ from the local ISM anti-apex direction) and from the "apex" sector (the part of the HSTOF field-of-view not included in the heliotail sector) measured by CELIAS/HSTOF ( $1 \sigma$ error bars). The accidental coincidence background is also shown.

concentration of the ions in the heliotail (Czechowski et al. 2001a). An alternative explanation is presented in Kota et al. (2001).

Only the low energy data points (taken here to correspond to the three lowest energy bins) can be reliably assumed to represent the ENA. For higher energy, ion contamination will be large. The higher-energy data points can only be regarded as upper limits on the ENA flux.

For helium, the ENA measurements are more difficult because of contamination by $\mathrm{He}^{2+}$ and $\mathrm{He}^{+}$ions. To estimate the ENA flux for helium it is necessary to assume the form of the spectra of the helium ions. The ENHe flux from the apex direction is close to the accidental background level (Fig. 2).

The upper limits on the heliospheric ENA fluxes were also reported by HENA (High Energy Neutral Atoms imager) on the IMAGE (Imager for Magnetopause-to-Aurora Global Exploration) satellite (Roelof, presentation at the COSPAR Colloquium The Outer Heliosphere: the New Frontiers, Potsdam 24-28 July 2000; Roelof et al., poster presented at 

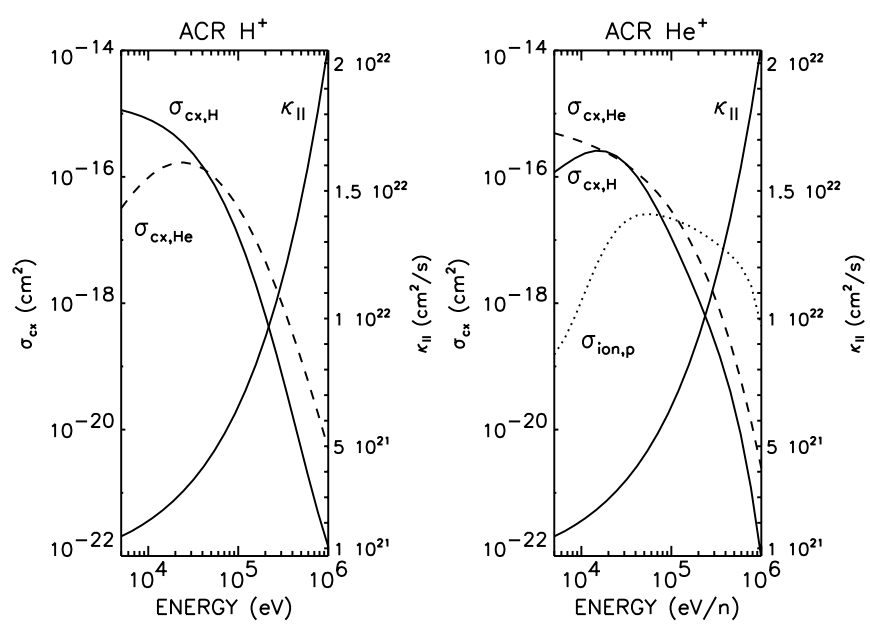

Fig. 3. Charge-exchange cross sections for $\mathrm{H}^{+}$(left) and $\mathrm{He}^{+}$(right panel) with neutral $\mathrm{H}\left(\sigma_{\mathrm{cx}, \mathrm{H}}\right)$ and $\mathrm{He}\left(\sigma_{\mathrm{cx}, \mathrm{He}}\right)$ atoms and the parallel diffusion coefficients $\kappa_{\|}$(right-side scale) assumed in the model. For $\mathrm{He}^{+}$the cross section for ionization $\mathrm{He}^{+} \rightarrow \mathrm{He}^{2+}$ by collision with protons is also shown $\left(\sigma_{\text {ion,p }}\right)$.

Solar Wind 10, Pisa, Italy, 2002; Roelof 2004, private communication). The energy range covered extends from $10 \mathrm{keV}$ to $50 \mathrm{keV}$ : that is, the upper energy region overlaps the HSTOF helium data. The implications of the HENA data will be discussed in Sect. 3.6.

\section{ACR termination shock spectrum from observations of the ENA}

\subsection{Relating the ENA flux to the ACR flux at the termination shock}

The following considerations are based on the assumption that the observed ENA derive predominantly from the ACR ions, and that the source region of the ENA is situated downstream from the termination shock. Other possible sources of the ENA in the HSTOF energy range were considered:

(1) ions accelerated at the interplanetary shocks associated with the co-rotating interaction regions (Hsieh et al. 1992; Giacalone et al. 1997; Kota et al. 2001). The ENA source region would then be situated in the inner heliosphere, where these structures are pronounced.

(2) pick-up ions accelerated by the turbulence in the supersonic solar wind (Chalov et al. 1995; Fahr \& Lay 2000) and subsequently transported downstream, with some energy gain on crossing the shock. The ENA could then originate both inside and outside the termination shock.

Theoretical estimations of the ENA flux for case (1) (Kota et al. 2001) are lower than the HSTOF data although this result is subject to model uncertainties. For case (2) an ENA flux as high as measured by HSTOF was obtained in one particular model (Czechowski et al. 2001c) but the energy spectrum was steeper than observed. We conclude that, although a large contribution to the observed ENA flux from other sources cannot be excluded, the possibility that the ENA come predominantly from the ACR ions can be taken as a working hypothesis.
The ACR distribution in the outer heliosphere and the associated ENA flux can at present only be derived from numerical solutions of the ACR transport equation, assuming some model of the heliospheric plasma flow and magnetic field structure. Our approach here is one of maximum simplification. We assume that (1) the plasma flow is constant in time (2) adiabatic acceleration and drift of the low-energy ACR ions can be neglected downstream of the termination shock (3) spatial diffusion of the ACR can be neglected or treated approximately (see below). The cross sections (Barnett et al. 1990) and the diffusion coefficients (le Roux et al. 1996) assumed in the following are shown in Fig. 3.

Disregarding the complications caused by time-dependence and possible asymmetry of the heliosphere, there are two special choices of viewing direction for which the ENA-ACR relationship becomes particularly simple: the heliospheric stagnation point (LISM apex) and the heliotail (LISM anti-apex) directions. The plasma flow is then parallel to the line of sight, and the sunward-directed ACR intensity downstream of the termination shock can be written in the form

$J_{\mathrm{ACR}}(\eta, E)=\left.J_{\mathrm{ACR}}(E)\right|_{\text {shock }} \mathrm{e}^{-\int_{0}^{\eta} \mathrm{d} \eta \beta(E, \eta) / V(\eta)}$

where $\eta$ is the distance downstream from the shock along the line of sight, $\beta$ the ACR loss rate from the line of sight and $V$ the plasma speed. The loss rate $\beta$ includes the charge-exchange rate $\beta_{\mathrm{cx}}=\left(n_{\mathrm{H}} \sigma_{\mathrm{cx}, \mathrm{H}}+n_{\mathrm{He}} \sigma_{\mathrm{cx}, \mathrm{He}}\right) u$ ( $u$ is the particle velocity) along with other terms: $\beta=\beta_{\mathrm{cx}}+\Delta$. The ACR ENA flux at the observation point in the inner heliosphere is given by the integral over the source region $\left(0, \eta_{\max }\right)$ along the line of sight

$J_{\mathrm{ENA}}(E)=\int_{0}^{\eta_{\max }} \mathrm{d} \eta \frac{\beta_{\mathrm{cx}}(E, \eta)}{u} J_{\mathrm{ACR}}(\eta, E)$.

Extinction is omitted since it is unimportant.

The loss rates $\beta$ and $\beta_{\mathrm{cx}}$ and the plasma velocity $V$ in the heliotail we take to be approximately constant over the source region. In the apex direction we take the flow speed to fall linearly between the termination shock and the stagnation point: $V(\eta)=V_{0}(1-\eta / L)$ where $L$ is the distance from the shock to the stagnation point. Substituting Eqs. (1) into (2) one then obtains

$\left.J_{\mathrm{ENA}}(E)\right|_{\text {tail }}=\left.J_{\mathrm{ACR}}(E)\right|_{\text {shock,tail }} \frac{V}{u} \frac{\beta_{\mathrm{cx}}(E)}{\beta(E)}$

for the heliotail direction $\left(\eta_{\max }=\infty\right)$ and

$\left.J_{\mathrm{ENA}}(E)\right|_{\mathrm{apex}}=\left.J_{\mathrm{ACR}}(E)\right|_{\text {shock,apex }} \frac{V_{0}}{u} \frac{\beta_{\mathrm{cx}}(E)}{\beta(E)+V_{0} / L}$

for the apex direction $\left(\eta_{\max }=L\right)$.

\section{2. "No-diffusion" and "leaky-tail" approximations}

While for moderate-to-high energy ACR (1-100 MeV) spatial diffusion is the dominant effect shaping their distribution, the situation is different for the lowest-energy end $(\sim 100 \mathrm{keV})$ of the ACR spectrum. Apart from the diffusion coefficients being smaller at lower energy, the loss of ions to charge-exchange becomes an important factor. Since these particles cannot penetrate far upstream from the termination shock, the low-energy 
ACR population is restricted to the outer heliosphere, and suppressed in the modulated ACR spectrum measured in the inner solar system.

Spatial diffusion of the ACR particles occurs predominatly along the magnetic field. A complete model of the ACR distribution would have to take into account the heliospheric magnetic field, which is expected to have a complicated, significantly three-dimensional and time-dependent structure (Nerney et al. 1991, 1993, 1995). For our choice of viewing directions, the local magnetic field would be approximately perpendicular to the line of sight.

Numerical solutions of the low-energy ACR transport equation in the outer heliosphere are at present available only for simplified (axisymmetric heliosphere, scalar approximation for the ACR diffusion) models (Czechowski et al. 1995, 2001a). The results imply that the low-energy ACR ions concentrate in the heliotail. Although this conclusion cannot be taken for granted (it could be invalidated if the ACR density at the termination shock is strongly suppressed in the heliotail direction) it is consistent with the HSTOF ENA data, which show a peak from the heliotail direction.

The heliotail is characterized by a relatively uniform, fast $\left(\sim 100 \mathrm{~km} \mathrm{~s}^{-1}\right)$ plasma flow. If the presently available approximate models (Czechowski et al. 2001a) can be taken as a guide, the ACR distribution inside the heliotail would, at low enough energy, be determined by convection and loss to charge-exchange. At higher energy the charge-exchange rate falls sharply and spatial diffusion becomes more important, in particular as a mechanism of escape of the ACR ions towards the boundary of the heliotail. The energy range in which the diffusion effects are small would depend on the relative values of the charge-exchange loss rate and the escape rate, the latter determined by the spatial diffusion tensor.

For low-energy ACR ions in the outer heliosphere we do not have a reliable estimate of the diffusion tensor. $\kappa_{\|}$shown in Fig. 3 (based on a low-energy extrapolation of a phenomenological formula derived for high-energy ions: le Roux et al. 1996) corresponds to a parallel mean free path $\lambda_{\|}$of $\sim 3 \mathrm{AU}$. In the heliotail, spatial diffusion effects would then be unimportant at $\sim 10 \mathrm{keV} / \mathrm{n}$ but not at $\sim 100 \mathrm{keV} / \mathrm{n}$ (Czechowski et al. 2001a). Since the value of $\kappa_{\|}$is uncertain, we cannot exclude the possibility that spatial diffusion can be neglected in the whole HSTOF ENA energy range ( 30-90 keV/n).

In consequence, we consider two cases:

- $\beta=\beta_{\mathrm{cx}}$ ("no-diffusion" approximation). Spatial diffusion and adiabatic acceleration of the ACR are disregarded and charge-exchange is assumed to be the only loss process of the ACR in the considered energy range.

$-\beta=\beta_{\mathrm{cx}}+\Delta, \Delta(E) \sim \kappa_{\|}(E)$ ("leaky-tail" approximation). The term $\Delta(E)$ describes approximately the escape of ACR particles by spatial diffusion towards the boundary of the heliotail. Specifically, we write

$\Delta(E)=C \kappa_{\|}(E)(\pi / 2 D)^{2}$

where $C$ is a free parameter and $D$ is the characteristic radius of the heliotail cross section. This expression is based on the approximate solution of the transport equation in the

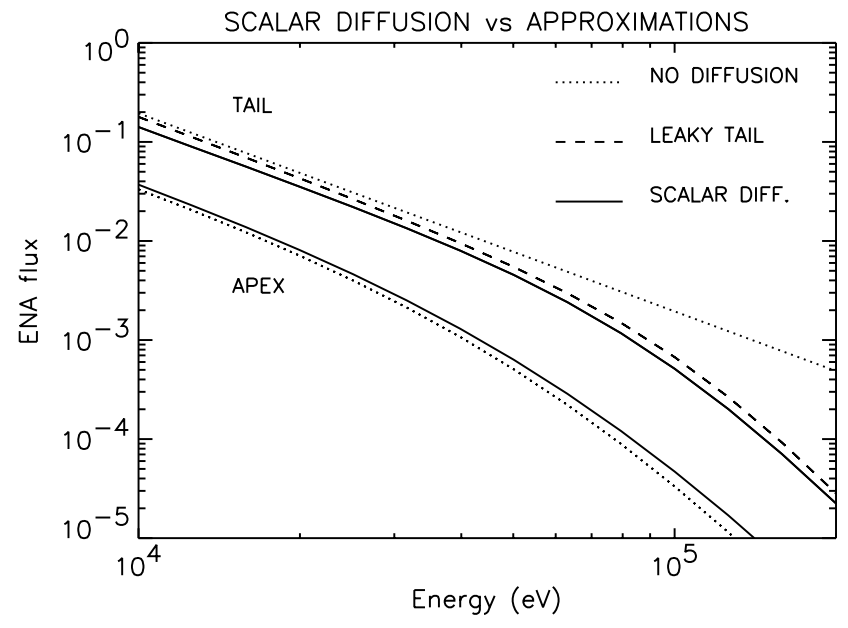

Fig. 4. No-diffusion and leaky-tail approximations compared to the scalar-diffusion model.

heliotail (Czechowski et al. 1995). For $C=1$ it can be obtained by assuming that diffusion is constrained to a single direction, perpendicular to the axis of the heliotail.

A test of these approximations is shown in Fig. 4. The ENA $\mathrm{H}$ fluxes following from "no-diffusion" and "leaky-tail" approximations are compared to the ENA spectra calculated from the ACR distribution derived by solving numerically the ACR transport equation in a model heliosphere, with ACR diffusion treated in the scalar approximation (Czechowski et al. 2001a). Besides spatial diffusion, the scalar-diffusion model included also other features neglected in the tested approximations, in particular adiabatic acceleration of the ACR ions; also, the "apex" and "tail" ENA spectra were defined as averages over wide angular sectors, as in the case of the HSTOF data (see Sect. 2). The "no-diffusion" and "leaky-tail" approximations are, however, in a reasonable agreement with the scalardiffusion model (for the tail case, the "no-diffusion" approximation is good below $\sim 50 \mathrm{keV}$ ).

\subsection{First-choice parameters}

The parameters of the ENA source region required by the above approximations are (first-choice values are given in brackets):

- "no-diffusion", tail: $V\left(100 \mathrm{~km} \mathrm{~s}^{-1}\right)$;

- "no-diffusion", apex: $V_{0}\left(100 \mathrm{~km} \mathrm{~s}^{-1}\right), L(42.5 \mathrm{AU}), n_{\mathrm{H}, \mathrm{apex}}$ $\left(0.125 \mathrm{~cm}^{-3}\right), n_{\text {He,apex }}\left(0.015 \mathrm{~cm}^{-3}\right)$;

- "leaky-tail": $V\left(100 \mathrm{~km} \mathrm{~s}^{-1}\right), \kappa_{\|}$(as in Fig. 3), $D$ (200 AU), $n_{\mathrm{H} \text {,tail }}\left(0.05 \mathrm{~cm}^{-3}\right), n_{\mathrm{He}, \text { tail }}\left(0.025 \mathrm{~cm}^{-3}\right)$.

The most reliable estimate is for $n_{\mathrm{He} \text {,apex }}$ (Witte et al. 2004). For $n_{\mathrm{H} \text {,apex }}$, values of $0.12-0.17 \mathrm{~cm}^{-3}$ follow from interpretations of Ly- $\alpha$ (Quemerais et al. 1994) and pick-up ion (Gloeckler et al. 1997) data; see also Izmodenov et al. (1999). The probable range of the post-shock plasma speeds $V$ and $V_{0}$ is $\sim 50-150 \mathrm{~km} \mathrm{~s}^{-1}$. L, D and the neutral densities in the heliotail are rough estimates based on numerical simulations of the heliosphere (Zank 1999; Fahr et al. 2000). In the following, the first-choice values are used unless otherwise specified. 
The parameter values may vary with time, in particular with the phase of the solar cycle. Using a time-dependent model of the heliosphere (K. Scherer, private communication) we find that the largest variation occurs for $L(\sim 30 \%$ of the maximum value). The changes in the remaining parameters are of the order of $10 \%$ or less. For these estimations, $V$ was taken to be defined as the average over the distance of $100 \mathrm{AU}$ downstream from the termination shock in the heliotail direction.

\subsection{Two-parameter fit using only HSTOF data}

We restrict the fits to the three lowest-energy data points, since we expect the higher-energy data to contain a significant contribution from ambient ions. As the considered energy interval is narrow, we assume that the parent-ion spectrum can be approximated by a pure power law, so that it is completely specified by two parameters: the flux intensity $J_{\text {ref }}$ at the reference energy $E_{\text {ref }}($ here $100 \mathrm{keV} / \mathrm{n})$ and the power $\gamma:\left.J_{\mathrm{ACR}}(E)\right|_{\text {shock }}=$ $J_{\text {ref }}\left(E / E_{\text {ref }}\right)^{-\gamma}$.

Figure 5 shows the results of the two-parameter $\chi^{2}$ fit using the "no-diffusion" approximation and first-choice parameters. We use $\chi^{2} \equiv \Sigma_{i=1}^{3}\left(F_{i}-f_{i}\right)^{2} / \delta_{i}^{2}$ where $F_{i}$ are the measured ENA flux values at energy $E_{i}, f_{i}$ the model predictions averaged over the energy bins and $\delta_{i}$ the experimental errors. The $\chi^{2}=3$ and $\chi^{2}=1$ contours are shown, both for the heliotail and the apex directions. Note that while the fits to the tail data admit reasonable solutions for the parent ion spectrum $(\gamma=1-2)$, for the apex helium data $\gamma<0$ is favoured, and for the apex hydrogen data it is not possible to obtain a good $\left(\chi^{2} \leq 1\right)$ fit. The difficulties with extracting the ENA spectrum from the "apex" data are most likely due to the low signal-to-noise ratio for this case (Figs. 1 and 2).

The ratio of the ENA flux from the heliotail to that from the "apex" sector is $\sim 2-3$ (Figs. 1 and 2). For the ENA of ACR origin, a higher ratio $(\sim 10)$ is expected (Fig. 4), assuming that the parent ion flux at the termination shock is approximately the same in the "apex" and in the "tail" regions. The higher-thanexpected ENA flux from the "apex" direction may indicate that the ACR flux at the termination shock is higher in the apex region. Another possibility is that, besides the ENA of ACR origin, the data include also another component, not peaked in the "tail" sector. The extra component would constitute a larger fraction of the (lower flux) "apex" data than of the "tail" data. The "apex" data would then have to be interpreted as an upper limit on the ACR ENA flux (dotted line in Fig. 5).

\subsection{Inclusion of the high-energy ACR data}

The values of $\gamma$ obtained from the fit to the HSTOF data have a large uncertainty. The result can be much improved if the highenergy ACR data from Voyagers (Cummings et al. 2002) are used as a constraint on the normalization of the ACR termination shock spectrum. The idea is to combine the ACR flux intensity at the termination shock derived from HSTOF data with the high-energy value from Voyagers to obtain the average slope $\gamma$ between these two points.
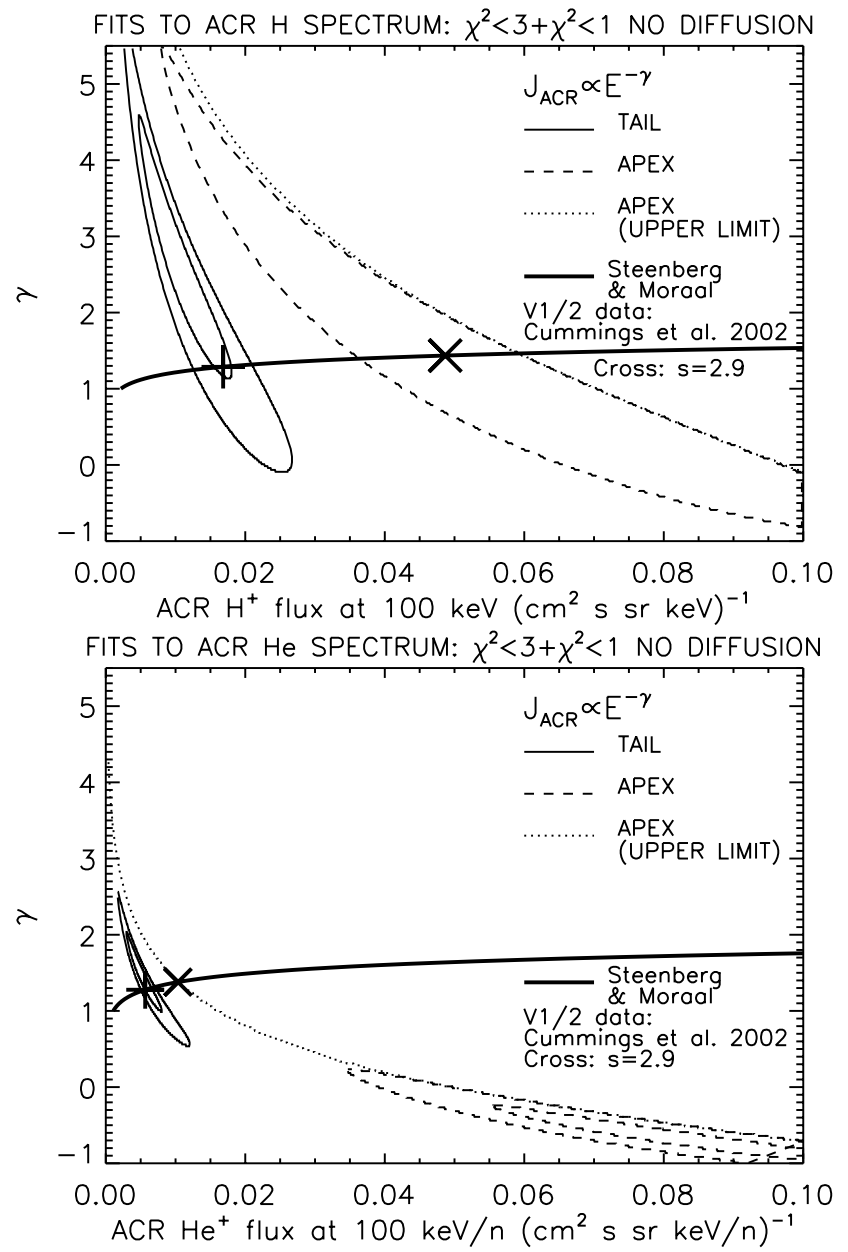

Fig. 5. Fits to HSTOF ENH and ENHe spectra in the "no-diffusion" approximation. The ACR spectrum at the termination shock in the energy range covered by the data is assumed to be of the form $J_{\text {ref }}\left(E / E_{\text {ref }}\right)^{-\gamma}$, where $J_{\text {ref }}$ is the ACR flux intensity at the reference energy $E_{\text {ref }}=100 \mathrm{keV} / \mathrm{n}$. Only the three lowest-energy data points are used in the fits. For the fits to the HSTOF data alone, $\gamma$ and $J_{\text {ref }}$ are treated as independent quantities (two-parameter fit). The $\chi^{2}=3$ and $\chi^{2}=1$ contours are shown, with solid (dashed) lines corresponding to the heliotail (apex) direction, respectively. For hydrogen, no $\chi^{2}<1$ region is found for the apex direction. The thick solid curve represents a constraint on $\left(\gamma, J_{\text {ref }}\right)$ obtained by requiring the ACR spectrum to agree with Voyager high-energy data, assuming the Steenberg \& Moraal form of the ACR shock spectrum. The best fits obtained under this requirement (the "restricted" fits) are indicated by the crosses (heliotail) and $X$ signs (apex). For the heliotail direction, the "restricted" fits give for both $\mathrm{H}$ and $\mathrm{He}$ the same value of $\gamma$ (corresponding to a shock compression ratio of 2.9) although the fits to the $\mathrm{H}$ and $\mathrm{He}$ data are independent.

One complication is that the power-law ACR shock spectrum is expected to break ("roll-off") at high energy. We assume that the ACR spectrum at the termination shock is given by the approximate formula of Steenberg \& Moraal (1999):

$\left.J_{\mathrm{ACR}}\right|_{\text {shock }}(E) \propto\left(E / E_{\mathrm{c}}\right)^{1-q / 2} \mathrm{e}^{-b\left(E / E_{\mathrm{c}}\right)^{a}}$

where $q=3 s /(s-1)$ and $s$ is the shock compression ratio. The remaining parameters: the "roll-off" energy $E_{\mathrm{c}}=$ $44 \mathrm{MeV}(\mathrm{H}), 11 \mathrm{MeV} / \mathrm{n}(\mathrm{He}), b=0.189$ and $a=2.029$ 
(Steenberg \& Moraal 1999) were chosen so that the resulting spectrum is close to the result of the de-modulation model of Cummings et al. (2002) for the weak-shock case. The normalization of the ACR flux at the shock is fixed by requiring that the flux is equal to $3.0\left(\mathrm{~m}^{2} \mathrm{~s} \mathrm{sr} \mathrm{MeV}\right)^{-1}$ at $130 \mathrm{MeV}$ for protons and $2.0\left(\mathrm{~m}^{2} \mathrm{~s} \text { sr MeV/n) }\right)^{-1}$ at $40 \mathrm{MeV} / \mathrm{n}$ for $\mathrm{He}^{+}$(values based on high-energy Voyager data: Cummings et al. 2002). In a more complete treatment, a separate form of the ACR spectrum would have to be used for the apex and the heliotail directions. The slope $\gamma=-1+q / 2$ of the low-energy part or, equivalently, the shock compression ratio $s$, is a free parameter to be determined by the fit. The relation between the slope $\gamma$ and the flux at reference energy $J_{\text {ref }}$ implied by the assumed form of the ACR spectrum defines a curve in the parameter plane $\left(J_{\text {ref }}, \gamma\right)$ (Fig. 5). If the ACR spectrum is given by Eq. (6) the fit to the ENA data should be restricted to this curve (the "restricted" fit). Two other curves (thin lines in Fig. 6, obtained by changing $E_{\mathrm{c}}$ to $E_{\mathrm{c}}^{+}$or $E_{\mathrm{c}}^{-}: E_{\mathrm{c}}^{+}=1.35 E_{\mathrm{c}}$ for $\mathrm{H}, 1.2 E_{\mathrm{c}}$ for $\mathrm{He}$, $E_{\mathrm{c}}^{-}=E_{\mathrm{c}} / 1.21$ for $\mathrm{H}, E_{\mathrm{c}} / 1.12$ for $\mathrm{He}$ ) are shown to give an idea of the associated uncertainty. Changing $E_{\mathrm{c}}$ to $E_{\mathrm{c}}^{+,-}$corresponds to varying the ACR flux at the original $E_{\mathrm{c}}$ by a factor of 2 up and down.

\subsection{Results}

An interesting result is that the best "restricted" fit values for $\gamma$ in the "no-diffusion" approximation to the heliotail ENA spectrum (crosses in Fig. 5) are almost exactly the same for $\mathrm{H}$ and $\mathrm{He}(\gamma \approx 1.28)$. This is what one should expect if the ACR spectrum is determined by diffusive shock acceleration, because $\gamma$ is then uniquely given for all ion species by the shock compression ratio $s: \gamma=(2+s) / 2(s-1)(s=2.9$ for $\gamma=1.28)$. If the (sole) parameter $V$ defining the "no-diffusion" approximation for the case of the heliotail is varied, the agreement between the results obtained for $\mathrm{H}$ and $\mathrm{He}$ persists (a consequence of the power-law behaviour of the ACR spectrum in the considered energy range), although the result for $\gamma$ depends on the value assumed for $V: \gamma=(1.38,1.29,1.21)$ corresponding to $s=(2.7,2.9,3.05)$ for $V=(50,100,150) \mathrm{km} \mathrm{s}^{-1}$, respectively. However, if the "leaky-tail" approximation $(C \neq 0$ in Eq. (5)) is used in place of the "no-diffusion" approximation, the best "restricted" fit values of $\gamma$ for $\mathrm{H}$ and He become different if the same value of $C$ is assumed for $\mathrm{H}$ and $\mathrm{He}$ (Fig. 6).

In distinction to the case of "restricted" fit, the best fit $\gamma$ values for the "no-diffusion" fits to the HSTOF heliotail data alone are different for $\mathrm{H}(\gamma=2.76)$ and $\mathrm{He}(\gamma=1.51)$. Only the helium value is close to what would be expected for the ACR termination shock spectrum. Since the energy per nucleon is lower for ENHe than for ENH data, there is a possibility that the "nodiffusion" approximation works better for helium. The differences in slope in the $\mathrm{H}$ and He heliotail ENA spectra could then be due to escape of the ACR ions from the line-of-sight, the effect taken into account in the "leaky-tail" approximation. In this case, the difference between the best fit values of $\gamma$ for $\mathrm{H}$ and $\mathrm{He}$ should be minimized by an appropriate choice of $C$ (Eq. (5)). Figure 6 shows the results of the "leaky-tail" fit with different values of the coefficient $C$ (the weight of the escape term). If $C$ for $\mathrm{H}$ and $\mathrm{He}$ is to be the same, there is no solution

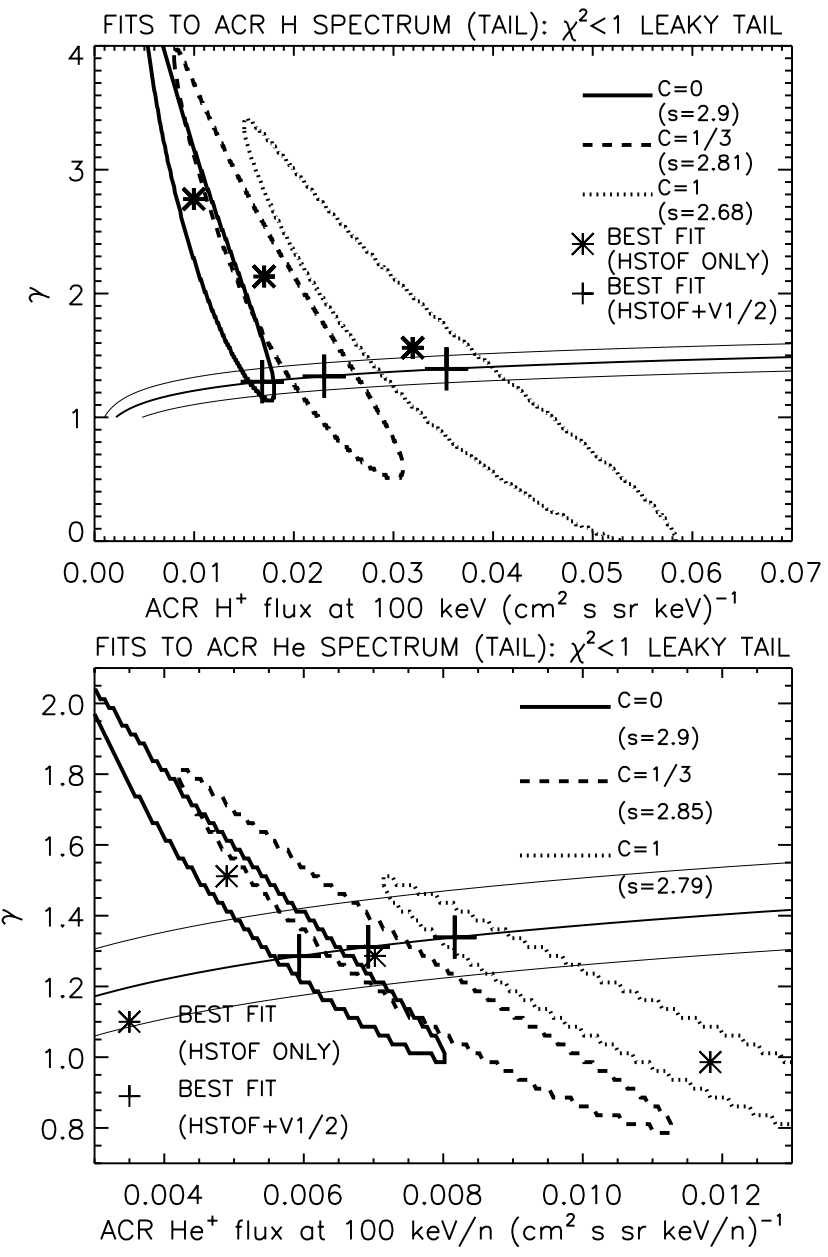

Fig. 6. Fits to the heliotail ENA spectrum in the "leaky-tail" approximation. The $\chi^{2}=1$ contours are shown. For each choice of $C$ (the weight of the escape term, Eq. (5)) the best fit obtained from HSTOF data alone is shown by an asterisk and the best fit restricted to the Steenberg \& Moraal ACR spectrum (thick solid line, with two thin lines showing the uncertainty) by a cross (the values of the shock compression ratio $s$ given in the plot are for the restricted fit). Note that the best fits to the HSTOF data alone can be made to agree with the "restricted" fits for specific choices of $C$ that are different for $\mathrm{H}$ and $\mathrm{He}$.

with the same best fit value for $\gamma$ for $\mathrm{H}$ and He. To obtain a solution it is necessary to modify the "leaky-tail" model by increasing the ratio of $\mathrm{H}$ and $\mathrm{He}$ escape terms $\Delta_{\mathrm{H}} / \Delta_{\mathrm{He}}$ by a factor of 4 or more. However, for the HSTOF data the statistics is not good enough to make this conclusion meaningful.

If the "apex" data are considered as an upper limit on the ACR ENA flux, the above results are consistent with the "apex" data. We have also considered the possibility that the "apex" ENA data are mostly of ACR origin. The shape of the ENA spectrum cannot be reliably determined from the "apex" data alone. Therefore we have considered a "restricted" fit, assuming that the shape of the ENA spectrum is determined by the ACR spectrum given by the Steenberg \& Moraal formula. Since $n_{\mathrm{He}} / n_{\mathrm{H}}$ is small $(\sim 0.1)$ in the apex direction, the no-diffusion approximation for this case depends effectively on two parameters: $n_{\mathrm{H}} L / V_{0}$ and $V_{0}$. For the first-choice parameters, the result is shown in Fig. 5 ( $X$ signs $)$ and Fig. 7. Varying 

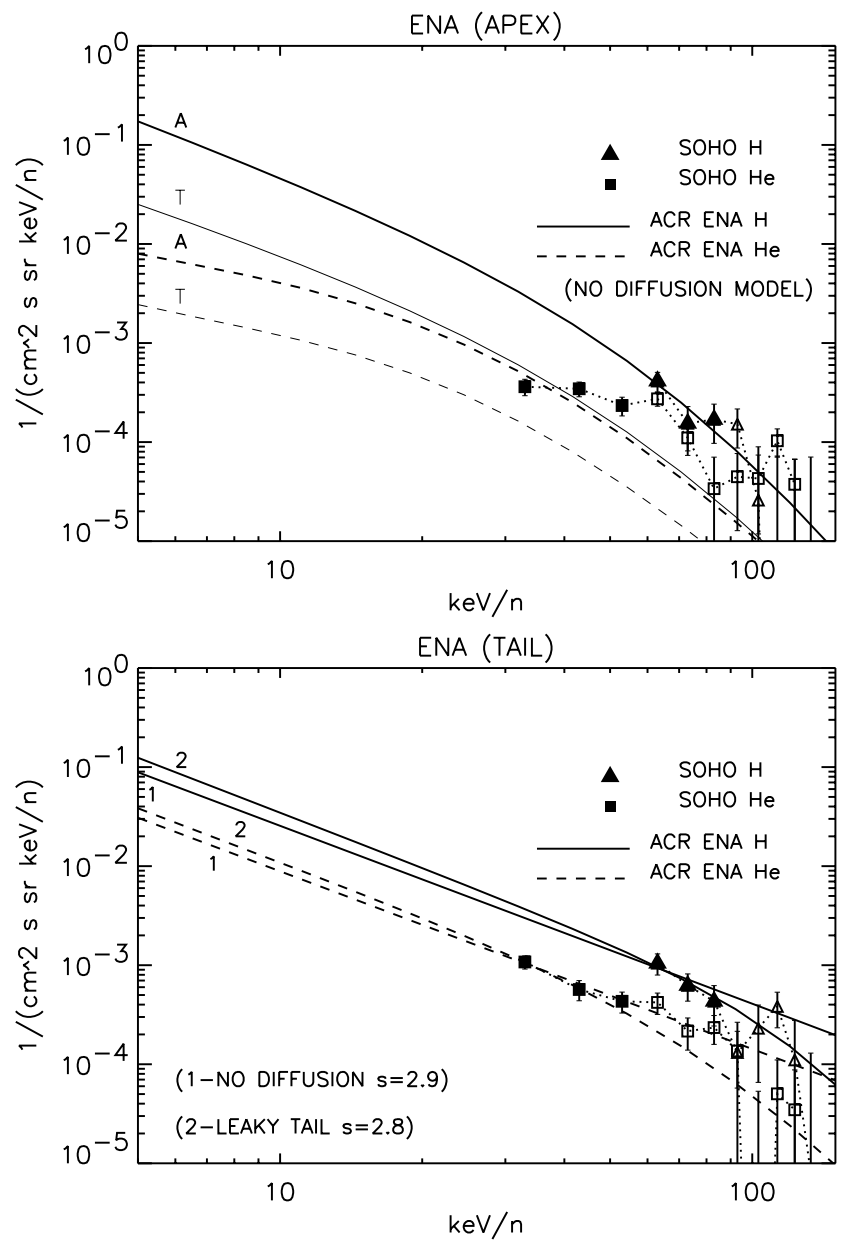

Fig. 7. ENA spectra for apex and tail direction resulting from the "restricted" fits compared to the HSTOF data. The curves (T) (upper panel) and (1) (lower panel) correspond to the same ACR spectrum at the shock, derived from the fit to the "tail" data in the "no-diffusion" model (first-choice parameters, $s=2.9$ ). (A) is derived from the fit to the "apex" data for the "no-diffusion" case. (2) is obtained from the fit to the "tail" data using the "leaky-tail" approximation $(s \approx 2.8$, $C=1 / 3$ for $\mathrm{H}, C=1$ for $\mathrm{He}$ ).

the parameters does not significantly improve the quality of the fits, which $\left(\chi^{2} \sim 1\right.$ for $\mathrm{H}$ and $\sim 10$ for $\left.\mathrm{He}\right)$ is lower than for the heliotail case $\left(\chi^{2}<1\right)$.

Figure 7 shows the ENA spectra derived from the ("restricted") fits to the heliotail and the apex data, respectively, compared to the HSTOF data points. The HENA/IMAGE data are still not available for publication. However, we compared the data to the preliminary upper limits from HENA/IMAGE (Roelof 2004, private communication) as presented at the 3rd International IGPP Conference "Physics of the Outer Heliosphere", Riverside, 2004. Since the HSTOF "heliotail" sector covers only a small part of the sky, it is the "apex" ENA data that should be compared to HENA. The HSTOF ENA data are consistent with these upper limits. The ACR ENA model is consistent with the HENA upper limits, provided that the ACR flux at the shock is derived from the "tail" HSTOF data, with the "apex" data regarded as upper limits on the ACR ENA flux.

\section{Discussion}

Assuming that the ENA detected by HSTOF are of ACR origin, we used approximate relations between the ACR spectra at the termination shock and the spectra of the ENA arriving in the inner solar system to obtain information about the ACR spectrum at the shock. The approximate relations, valid for special choices of viewing direction (apex and heliotail), do not require detailed knowledge of the outer heliosphere.

Because of the large statistical errors and narrow energy range of the HSTOF ENA data, the parameters of the ACR spectrum derived from the HSTOF data alone have a large uncertainty. Much better results follow when Voyager 1/2 highenergy ACR data are used as a constraint on the normalization of the ACR spectrum.

The main result is that, if the observed ENA come from ACR ions, the ACR spectrum must be steep, implying a weak (compression ratio below 3) termination shock. A weak termination shock is in fact indicated by theoretical simulations (Baranov \& Malama 1993; Pauls \& Zank 1997; Linde et al. 1998; Zank 1999; Fahr et al. 2000) and consistent with ACR observations by Voyagers (Cummings et al. 2002). The $\mathrm{He} / \mathrm{H}$ ENA ratio as measured by HSTOF is consistent with the highenergy ACR data (Cummings et al. 2002).

One conclusion that emerges from our study is the importance of measuring the ENA flux in a wide energy range. The "No-diffusion" approximation, for which dependence on unknown outer-heliospheric parameters is much reduced, is probably valid below $\sim 40-50 \mathrm{keV} / \mathrm{n}$. On the other hand, the ACR spectrum at the termination shock is expected to simplify to a shock-strength-determined power law above the injection energy ( 100-200 keV/n). Those energy regions could be more appropriate for the approximate methods described in the preceding section than the energy interval covered by the HSTOF ENA data.

We have concentrated on the possibility that the observed ENA come from ACR ions. One reason for this is that this is the only case for which the uncertainty in the data can be reduced by using Voyager results. Although our results give some support to the ACR case (the same shock parameters obtained for both hydrogen and helium) the possibility of other sources of the observed ENA, like ions accelerated in the supersonic solar wind either by transient shocks (Hsieh et al. 1992; Giacalone et al. 1997; Kota et al. 2001) or by plasma turbulence (Chalov et al. 1995; Fahr \& Lay 2000; Czechowski et al. 2001c), cannot be excluded. If the contribution to ENA from these sources is shown to be high, the problem of separating the ACR ENA signal may become difficult, particularly near the ecliptic. With good statistics it should, nevertheless, be feasible to deconvolve the different contributions to the observed ENAs along the line of sight. If the time-stationary "no-diffusion" approximation is applicable, a signature of the ENA of ACR origin (and, more generally, of the ENA originating downstream from the termination shock from the ions that were accelerated upstream from the ENA source region) would be the difference in shape between the ENA energy spectrum from the heliotail and the ENA spectra from other directions: the heliotail ENA spectrum would, apart from the factor $E^{-1 / 2}$, follow the shape of the 
parent ion spectrum, while the spectra from other directions would be significantly steeper at higher energy due to energy dependence of the charge-exchange rate.

Our results were derived using a time-stationary picture. The heliospheric parameters and the ACR spectrum at the termination shock are expected to be time-dependent. The timevariation of the ENA flux would, however, be reduced due to the fact that the ENA source region extends over a large distance. The plasma parcel leaving the termination shock would contribute to the observed ENA flux for the time $1 / \beta$ (the ion loss time) or until it leaves the line of sight. Consequently, the observed ENA spectrum would be sensitive to the ACR spectrum at the shock averaged over this period of time. For the ENA in the HSTOF energy range our estimate for the chargeexchange loss time $1 / \beta_{\mathrm{cx}}$ is of the order $\sim 5 \times 10^{8} \mathrm{~s}$ for $\mathrm{H}$ and $\sim 2.5 \times 10^{8} \mathrm{~s}$ for He. In the no-diffusion model $\left(\beta \approx \beta_{\mathrm{cx}}\right)$ this implies that the ENA spectrum from the heliotail should be approximately constant below this time scale. The HSTOF data indicate that the hydrogen ENA flux from the tail may be timevariable on the scale of few $(\sim 5)$ years, however, because of low statistics caused in part by observational difficulties during the active Sun period, this result is not conclusive (not significant at the 3 sigma level). If the time variation on the scale of a few years is confirmed, this would indicate either that we have underestimated the charge-exchange rate, or that chargeexchange is not the dominant loss mechanism.

To summarize, we have demonstrated, using existing data from $\mathrm{SOHO}$, how energetic neutral $\mathrm{H}$ and He fluxes measured at $1 \mathrm{AU}$ can be used to derive the anomalous cosmic ray spectrum at and the shock strength of the termination shock. A necessary assumption is that the observed ENA are of ACR origin. To take full advantage of the method would require widening the ENA energy window from that of the existing HSTOF/CELIAS/SOHO to 30-300 keV. A description of such an instrument dedicated to heliospheric ENA measurements is presented in Hsieh et al. (2004).

Acknowledgements. A.C. wishes to thank the Max-Planck-Institut fuer Aeronomie for hospitality and M.H. thanks for support by the INTAS Grant WP 270. The work done at the University of Arizona is funded by NSF grant ATM 0108160 and NASA grant NAG5 11596.

\section{References}

Baranov, V. B., \& Malama, Yu. G. 1993, J. Geophys. Res., 98, 15157 Barnett, C. F., Hunter, H. T., Kirkpatrick, M. I., Alvarez, I., \& Phaneuf, R. A. 1990, Atomic Data for Fusion. Collisions of H, H2, He and Li Atoms and Ions with Atoms and Molecules, Oak Ridge Natl. Lab. Report, ORNL-6086-VI, Oak Ridge, Tenn.

Burlaga, L. F., Ness, N. F., Stone, E. C., et al. 2003, Geophys. Res. Lett., 30, 2072
Chalov, S. V., Fahr, H. J., \& Izmodenov, V. 1995, A\&A, 304, 609

Cummings, A. C., Stone, E. C., \& Steenberg, C. D. 2002, ApJ, 578, 194

Czechowski, A., Fichtner, H., Grzedzielski, S., et al. 2001a, A\&A, 368,622

Czechowski, A., Hilchenbach, M., \& Hsieh, K. C. 2001b, in Proc. of the 27th International Cosmic Ray Conference, Hamburg, August 2001, 10, 4227

Czechowski, A., Fahr, H. J., Lay, G., \& Hilchenbach, M. 2001c, A\&A, 379,601

Czechowski, A., Hsieh, K. C., Hilchenbach, M., Kota, J., \& Shaw, A. W. 2004, Adv. Space Res., 34, 104

Czechowski, A., Grzedzielski, S., \& Mostafa, I. 1995, A\&A, 297, 892

Fahr, H. J., \& Lay, G. 2000, A\&A, 356, 327

Fahr, H. J., Kausch, T., \& Scherer, H. 2000, A\&A, 357, 268

Giacalone, J., Jokipii, J. R., Decker, R. B., et al. 1997, ApJ, 486, 471

Gloeckler, G., Fisk, L. A., \& Geiss, J. 1997, Nature, 386, 374

Gruntman, M. A. 1992, Planet. Space Sci., 40, 439

Gruntman, M., Roelof, E. C., Mitchell, D. G., et al. 2001, J. Geophys. Res., 106, 15767

Hilchenbach, M., Hsieh, K. C., Hovestadt, D., et al. 1998, ApJ, 503, 916

Hilchenbach, M., Hsieh, K. C., Hovestadt, D., et al. 2001, in The Outer Heliosphere: The New Frontiers, COSPAR Colloquium Series, 11,273

Hsieh, K. C., Shih, K. L, Jokipii, J. R., \& Grzedzielski, S. 1992, ApJ, 393, 756

Hsieh, K. C., Zurbuchen, T. H., Orr, J., et al. 2004, Adv. Space Res., 34,213

Izmodenov, V. V., Geiss, J., Lallement, R., et al. 1999, J. Geophys. Res., 104, A3, 4731

Kota, J., Hsieh, K. C., Czechowski, A., Jokipii, J. R., \& M. Hilchenbach 2001, J. Geophys. Res., 106, 24907

Krimigis, S. M., Decker, R. B., Hill, M. E., et al. 2003, Nature, 426, 45

le Roux, J. A., Potgieter, M. S., \& Ptuskin, V. S. 1996, J. Geophys. Res., 101, 4791

Linde, T. J., Gombosi, T. I., Roe, P. L., et al. 1998, J. Geophys. Res., 103,1889

McDonald, F. B., Stone, E. C., Cummings, et al. 2003, Nature, 426, 48

Nerney, S., Suess, S. T., \& Schmahl, E. J. 1991, A\&A, 250, 556

Nerney, S., Suess, S. T., \& Schmahl, E. J. 1993, J. Geophys. Res., 98 , 15169

Nerney, S., Suess, S. T., \& Schmahl, E. J. 1995, J. Geophys. Res., 100, 3463

Quemerais, E., Bertaux, J.-L., Sandel, B. R., \& Lallement, R. 1994, A\&A, 290, 941

Schwadron, N. A., \& McComas, D. J. 2003, Geophys. Res. Lett., 30, 1587

Steenberg, C. D., \& Moraal, H. 1999, J. Geophys. Res., 104, A11, 24879

Witte, M., Banaszkiewicz, M., Rosenbauer, H., \& McMullin, D. 2004, Adv. Space Res., 34, 61

Zank, G. P. 1999, Space Sci. Rev., 89, 413 